\title{
La pandemia de Covid-19 en México: el papel fundamental de los cuidados paliativos y de la bioética
}

\section{The Covid-19 pandemic in Mexico: The fundamental role of palliative care and bioethics}

\author{
Elvira Llaca García, * Luz Adriana Templos Esteban**
}

https://doi.org/10.36105/mye.2021v32n1.05

\section{Resumen}

La pandemia de Covid-19 ha puesto a prueba a todos los sistemas de salud. La crisis que provocó ha impactado a la sociedad, economía y, en general, a todas las actividades humanas. Los cuidados paliativos y la bioética tienen un papel fundamental en el manejo de la nueva enfermedad Covid-19, que no tiene aún tratamiento conocido. Los principios de la bioética, sumados a la práctica de los cuidados paliativos han sido el soporte de la pandemia. Los pacientes recuperados y aquellos muertos a causa del Covid-19 han requerido en algún momento de medidas de soporte y paliación, ya sea en unidades hospitalarias o en su domicilio. Es deseable que se impulsen estas herramientas: la paliación y la ética de la vida, ya que no sólo las soluciones que permiten cuidar la vida y la salud provienen de la tecnología, y no deben ser

\footnotetext{
* Colegio Mexicano de Cuidados Paliativos y Soporte. Facultad de Bioética. Universidad Anáhuac México, México. Correo electrónico: elvira.llacag@anahuac.mx https://orcid.org/0000-0002-7971-1811

** División de Cuidados Paliativos y Clínica del Dolor del Hospital General Dr. Manuel Gea González, Ciudad de México, México. Correo electrónico: luzadrianatem plos@hotmail.com https://orcid.org/0000-0001-9377-6964

Recepción: 20 de septiembre de 2020. Aceptación: 15 de octubre de 2020.
} 
E. Llaca García, L. A. Templos Esteban

mutuamente excluyentes. A un año del inicio de la pandemia, hay muchas preguntas por responder, mucha investigación por hacer. Sin duda, el año 2020 dará motivos de reflexión a todos los seres humanos y, en especial, a los responsables de cuidar la salud.

Palabras clave: pandemia, Covid-19, cuidados paliativos, bioética, soporte.

\section{Introducción}

La pandemia de Covid-19 es tema de muchos escritos, discusiones académicas, médicas y tema habitual en los medios de comunicación. Algunas voces proponen que existió una realidad global antes del surgimiento del virus en Wuhan, y existirá una nueva realidad después de él, o bien junto a él, cuando podamos convivir con el coronavirus sin riesgo a enfermar, porque ya hemos creado inmunidad, como históricamente ha sucedido con el surgimiento de los nuevos patógenos.

El presente artículo tiene la intención de resaltar dos herramientas que pueden trabajar muy bien juntas: los cuidados paliativos y la bioética, acompañando a los enfermos y a sus familias, y también a los profesionales de la salud quienes, para cumplir con la lex artis de su profesión, requieren apoyarse en diversos puntos de vista, desde la terapéutica al acompañamiento, siempre en un marco de valores.

Este artículo se divide en tres partes: la pandemia, los cuidados paliativos y la bioética. Presenta una visión desde el campo de batalla y desde la academia, que pretende resaltar la importancia de cultivar e incrementar la formación, información y preparación en cuidados paliativos y en bioética del personal de salud y de toda persona interesada en la medicina y la ética. 
La pandemia de Covid-19 en México: el papel fundamental de los cuidados...

\section{La pandemia de Covid-19}

En los últimos días del 2019, una catástrofe se gestó en Wuhan, China, y pocos meses después repercutió en el mundo entero, trastornando la vida del ciudadano posmoderno en los campos sanitarios, sociales, emocionales y económicos.

La causa de la crisis mundial que debilitó al hombre en constante empoderamiento, y sigue haciéndolo todavía un año después, proviene de un agente patógeno, de talla pequeñísima: de 65 a $125 \mathrm{~nm}$ de diámetro (1). Hoy se sabe que este virus es el SARS-CoV-2, compuesto de ARN. El ya muy estudiado miembro de la familia de los coronavirus tiene un $96 \%$ de similitud con el Bat-CoV. La enfermedad del SARS-CoV-2 fue nombrada Covid-19 por la Organización Mundial de la Salud. El ciclo de vida de este coronavirus ha implicado parasitar inicialmente a un murciélago y, posteriormente, a otro animal intermedio, para llegar finalmente al ser humano. Se considera una zoonosis (enfermedad que se trasmite en forma natural de los animales al ser humano) (2).

Una de las primeras pandemias en la historia de la que se tiene noticia es la «plaga de Justiniano», en el Imperio Romano de Oriente o Bizantino, y se extendió desde el año 541 hasta el 543 por Europa, Asia y África, cobrando la vida de 25 millones de personas. Los historiadores suponen que la causante fue la bacteria Yersinia pestis, mismo agente causal de la «peste negra», que en el siglo XIV mató a 75 millones de personas. Yersinia pestis es inyectada al ser humano por una pulga transportada por una rata. Actualmente la Yersinia pestis puede ser controlada médicamente con antibióticos.

En el siglo Xx, en 1918, después de la Primera Guerra mundial, la "gripe española», causada por la influeza del virus AH1N1, dejó unos 100 millones de muertos. En 2009, un virus del mismo tipo provocó nuevamente una pandemia de influeza $H 1 \mathrm{~N} 1$, en la que murieron 18.000 personas aproximadamente.

Todavía en el siglo XXI las enfermedades virales siguen sin tener tratamiento. El manejo de los padecimientos virales consiste en dar 
soporte al enfermo, en espera de que sus sistema inmune logre dominar la enfermedad (3).

El Covid-19 aún no está totalmente descrito; se requiere tiempo para poder hacer un estudio del pronóstico y de las posibles complicaciones en el mediano y largo plazo.

La severidad del cuadro clínico del Covid-19 es muy variable: asintomático, enfermedad leve, estable con o sin repercusión respiratoria, afectado e inestable pero no en estado crítico, críticamente enfermo. Esta descripción se hizo en los primeros meses de la pandemia en Italia, y hoy las condiciones no han cambiado mucho. La severidad de la afección y la posibilidad de poner la vida del enfermo en peligro dictará la modalidad del tratamiento (4).

\section{Cuidados paliativos}

Los cuidados paliativos surgieron con el hombre; en general, el enfermo es paliado en su dolor y sufrimiento por su propia familia y por sus allegados. Se sabe que en la Edad Media, durante la epidemia de la peste negra, la única medida posible era el soporte de los síntomas y el acompañamiento.

La historia formal de los cuidados paliativos inició a finales del siglo XIX en Europa. En algunos centros de población ubicados en el paso de los viajeros había centros de acogida para los enfermos moribundos (5).

En este sentido fue trascendente el inicio del movimiento «Hospice», ya que dio lugar a reconocer la necesidad de cuidar a los enfermos que no tenían curación. St Cristopher Hospice, a cargo de Cicely Sauders, fue fundado en Inglaterra en el siglo Xx y, posteriormente, el modelo se replicó en varios países (5).

En México, los cuidados paliativos fueron desplazados durante el siglo XX de las instituciones de salud por la tecnología aplicada al diagnóstico y tratamiento de las enfermedades. Hoy sabemos que la paliación y la tecnología biomédica no son mutuamente exclu- 
La pandemia de Covid-19 en México: el papel fundamental de los cuidados...

yentes; se necesitan ambas opciones: curar con todo lo existente, sin llegar a la distanasia, y cuidar cuando ya no es posible curar. El avance de los cuidados paliativos en México es importante; no son la solución para el enfermo oncológico, como lo fueron originalmente, sino la alternativa para mejorar la calidad de vida de personas terminales, con enfermedades crónicas y para pacientes enfermos, cuya evolución natural de la enfermedad es la curación sin la intervención de un tratamiento médico (6).

México se encuentra entre los pocos países con una legislación clara en cuidados paliativos. Éstos son un derecho sanitario de sus habitantes. Es prioridad fortalecer la formación en ellos, tener unidades para la atención de los pacientes que requieren paliación y favorecer las políticas públicas que apoyen esta importante área de la medicina (7).

La Organización Mundial de la Salud ha definido los cuidados paliativos como la manera de mejorar la vida del paciente y de su familia, en caso de padecer una enfermedad que amenaza la vida, atendiendo el dolor y los síntomas, y dando apoyo espiritual y psicológico, desde el diagnóstico del padecimiento hasta la muerte y durante el duelo (8). La unidad de cuidados paliativos acompaña al enfermo y a su familia, y una vez que el paciente muere, acompaña también a la familia en su duelo.

En 2020, la Comisión Lancet redefinió los cuidados paliativos tras un riguroso estudio, y llegó a la conclusión de que la definición de la OMS de 2002 se tornó insuficiente. Los cuidados paliativos de hoy incluyen la prevención, la identificación temprana, el manejo de los problemas físicos y otros síntomas como el distrés psicológico y espiritual, además del cuidado de las necesidades sociales. La intervención debe estar basada en evidencias. Proveen soporte y ayuda a la vida del paciente hasta cuando aparezca la muerte natural, facilitando la comunicación y siguiendo las metas del paciente. Se aplican siempre de acuerdo con las necesidades del paciente. Se proveen junto con otras medidas terapéuticas que necesite el enfermo. Son una influencia positiva en la enfermedad. No posponen la 
muerte, sino que afirman la vida en su final. Proveen soporte a la familia durante la enfermedad del paciente. Reconocen los valores culturales del enfermo y de su familia. Se aplican en casa, así como en instituciones y hospitales, y en nivel primario, secundario o de alta especialidad. Pueden ser brindados por profesionales con entrenamiento básico. Se requiere de equipos multidisciplinarios. Deben adoptarse políticas públicas desde el Gobierno que incluyan los cuidados paliativos. Los seguros médicos deben incluirlos. Requieren proporcionar acceso a medicamentos contra el dolor y la tecnología básica para el manejo de dolor. Deben ser parte de los servicios básicos de salud, e involucrar a población vulnerable y a niños. Las universidades, hospitales y lugares de formación de personal de salud deben incluir esta asignatura en su programa de estudios. El estudio de la Comisión Lancet se verifica durante la pandemia que estamos presenciando (9).

El Covid 19 ha puesto a prueba a todos los sistemas de salud, provocando una crisis sanitaria internacional, inicialmente por la necesidad creciente de camas de hospital, de ventiladores mecánicos, de insumos y por la insuficiencia de profesionales de la salud. A un año del inicio de la enfermedad, no se ha descrito un tratamiento efectivo, a pesar de la investigación en este campo. La paliación de dicha enfermedad para aliviar el sufrimiento es la mejor manera de ayudar a los enfermos y a sus familias (10).

Sabemos que el espectro clínico de la enfermedad es amplio: puede ser asintomático, con sintomatología moderada, o paciente con neumonía severa y falla orgánica múltiple.

Desde la esencia misma de la medicina paliativa y de soporte, son cardinales cuatro principios en la paliación de las personas infectadas por el SARS-Cov-2:

Principio 1. Aliviar los sintomas El enfermo con Covid-19 frecuentemente presenta disnea, que puede ser manejada con medicamentos o con medidas físicas. En el primer caso, se usará la mínima dosis de opioide o benzodiacepina, 
La pandemia de Covid-19 en México: el papel fundamental de los cuidados...

de manera que sea capaz de evitar la sensación de falta de aire a nivel central. En el segundo caso, se recolocará al paciente en su cama para que le sea menos difícil la respiración. El cuadro clínico suele estar acompañado de sufrimiento; de necesidades sociales, emocionales y espirituales que requieren atención y se dificultan, ya que el paciente debe estar aislado y la familia no puede acompañarlo. La paliación debe dirigirse al paciente y a su familia ya que, como sabemos, la unidad de cuidados paliativos es el enfermo y su familia, y aquí surge un nuevo reto para aliviar los síntomas (10).

\section{Principio 2. Cuidado centrado en el paciente}

Incluye la necesidad de tomar en cuenta siempre las preferencias del paciente. Básicamente, el paciente consciente debe decidir si acepta ser trasladado a un hospital, si quiere ser conectado a un ventilador $\mathrm{y}$, en caso de que el paciente ya no pueda decidir, se debe subrogar este derecho a sus familiares. En las circunstancias de esta pandemia, es necesario guardar un equilibrio entre el cuidado centrado en el paciente y el bien de la comunidad. Los médicos deberán comunicar al paciente y a su familia que la preferencia del paciente no puede cumplirse por el riesgo de contagio del virus causal de la enfermedad, por las comorbilidades del paciente $y$, en ocasiones, por la situación de los recursos (10).

\section{Principio 3. Cuidar al paciente y a la familia}

La enfermedad afecta al paciente, a su familia y allegados, y todos requieren cuidado. La comunicación es un aspecto fundamental en el manejo de los pacientes en todas las circunstancias. El indispensable aislamiento de la persona infectada con Covid-19 complica mucho el cumplimiento de este principio. Hemos visto a enfermos acompañados a través de tabletas electrónicas, de celulares con videollamada y, en general, de cualquier medio digital que permita la comunicación a distancia. Sin embargo, aunque es útil, no elimina totalmente la ansiedad de los enfermos y sus familias. Hoy vemos 
que la «videodespedida» es una triste realidad cuando el enfermo no puede estar acompañado en la muerte, como aparece en la fotografía que Francesca Cortellaro publicó en Milán.

Se ha incrementado la necesidad de establecer conversaciones difíciles con pacientes y familias, respecto del pronóstico, del triaje y sobre la falta de ventiladores en los medios hospitalarios. Esta habilidad es indispensable en cuidados paliativos (10).

\section{Principio 4. Tener equipos multidisciplinarios}

Las necesidades del enfermo y de sus familias surgen de la multidimensionalidad humana, y es necesaria la atención de todos los aspectos que surgen. Como en ninguna otra época, el personal de salud ha debido asumir otras funciones diferentes de las suyas, y durante esta pandemia ha sido característico el «entrenamiento cruzado» para adaptarse a las circunstancias hospitalarias y de manejo domiciliario. El especialista en medicina paliativa ha sido sobrepasado y más que nunca ha sido valorado en su actuación (10).

La medicina paliativa y los cuidados paliativos se ubican en la primera línea de servicio a la comunidad. Es recomendable mantener al enfermo en casa, con cuidados paliativos, y darle seguimiento cercano por medio de telemedicina o de llamadas telefónicas. El médico, en el caso de un enfermo con comorbilidades y estado de vulnerabilidad, debe decidir junto con el enfermo y la familia la conveniencia de mantenerlo en casa, limitando el riesgo de adquirir una infección hospitalaria y de estar aislado lejos de casa. En el manejo de todo enfermo siempre será conveniente conocer su voluntad anticipada y preguntarle sus preferencias para cuando él no pueda decidir (11).

Durante la pandemia se ha modificado la esencia de los cuidados paliativos, la cual radica en el acompañamiento y cercanía. El paciente debe estar aislado; las visitas familiares no existen; quienes se acercan al enfermo usan un equipo especial que da sensación de lejanía e irrealidad; en caso de haber otro enfermo en el cuarto de 
La pandemia de Covid-19 en México: el papel fundamental de los cuidados...

hospital, debe estar separado al menos dos metros, y el personal sanitario limitar sus visitas (12).

En cada unidad de atención médica y en el domicilio en donde debe prestarse ayuda a una persona con Covid-19, la pandemia requiere resolver los síntomas que presenta la persona enferma, además de su observación constante, para resolver oportunamente los problemas y complicaciones que se presenten y manejar sus síntomas. La Organización Mundial de la Salud, en su Guía sobre cómo mantener los servicios de salud durante la pandemia, habla de la inmunización (cuando esté disponible), de la atención materna, de la atención de emergencias y de las enfermedades crónicas, entre otras, pero se olvida de los cuidados paliativos, que son indispensables.

La disponibilidad de opioides y el conocimiento para recetarlos, la telemedicina, los planes de servicios anticipados, la capacitación del personal de salud y de los cuidadores primarios es esencial, y es el quehacer cotidiano de la medicina paliativa (13).

\section{Bioética}

La bioética es una interdisciplina que nació en 1970, desarrollada por Van Rensselaer Potter en Estados Unidos. Hoy, la interdisciplina de Potter se enfrenta por primera vez a una crisis sanitaria global: es la mayor prueba que la bioética ha enfrentado desde su nacimiento, y puede decirse que ha estado presente acompañando a los médicos y elaborando guías de actuación. Seguramente muchos ciudadanos globales con acceso a la información formal e informal han escuchado en repetidas ocasiones la palabra «bioética».

Reich define la bioética en 1978 como el estudio sistemático de la conducta bumana en el área de las ciencias de la vida y la atención de la salud, en tanto que dicha conducta es examinada a la luz de principios y valores bumanos (14).

La necesidad más urgente y emanada de la práctica médica de contar con una interdisciplina, la bioética, que se ocupara de la éti- 
ca en las intervenciones sobre el hombre, inició en 1947, cuando Claude Beck realizó la primera desfibrilación. Con esto logró lo que el hombre había soñado siempre: revertir la muerte. En 1950, Bower y Bennett desarrollan el primer ventilador mecánico de presión positiva, y en 1955 apareció en forma comercial el Bird Mark 7, el primer «respirador artificial», motivo de serios y nuevos problemas de tipo diagnóstico, pronóstico y ético, ya que la muerte cardiaca se separó de la cerebral, surgiendo en muchas ocasiones la pregunta acerca de la calidad de vida del enfermo. La crisis ética de aquellos que no podían ofrecer un ventilador a sus pacientes invitó al papa Pío XII a llamar «extraordinaria» a toda medida que estuviera fuera del alcance del médico o que tuviera mal pronóstico (15).

La sobrevaloración de la tecnología aplicada al manejo de las enfermedades caracteriza al mundo contemporáneo. La bioética balanceó la tecnología aplicable a la salud con el impacto de lo que significan los tratamientos para el enfermo, y ha insistido en el respeto a la autonomía del enfermo.

Actualmente, la extensión de la discusión bioética es enorme: va desde la creación de ciborgs hasta la necesidad de aplicar la justicia distributiva; desde el uso de cirugía robótica, hasta la medicina paliativa (16).

La salud pública siempre está influenciada por las decisiones políticas del momento, lo cual hace, en ocasiones, más complejo el diálogo indispensable entre los actores de la bioética. En la crisis sanitaria actual hemos visto que las palabras de Edmond Pellegrino son reales: la política interviene en todo momento en las decisiones sobre la salud (16).

En México, la epidemia de obesidad detectada desde 1988 ha traído como consecuencia el incremento de enfermedades metabólicas en la población, en todas las edades, y esto se debe, sin duda, a las políticas alimentarias del país. Hay una tendencia permanente al sobrepeso y a la obesidad, que ponen en evidencia la necesidad de establecer políticas públicas tendientes a regular los alimentos que se ofrecen a la población. Lamentablemente la obesidad inicia 
La pandemia de Covid-19 en México: el papel fundamental de los cuidados...

en la infancia y se ha normalizado en la sociedad mexicana, sin pensar que esto es fuente de diabéticos infantiles, de morbilidad alta y de deterioro de la salud (17). México recibe al Covid-19 en plena epidemia de obesidad, lo cual hace a esta población más susceptible de infectarse al tener una comorbilidad, ya que hace al enfermo más vulnerable. Durante la pandemia de Covid-19 se decide etiquetar los alimentos que tienen exceso en sodio, azúcar, grasas saturadas y grasas trans, sodio y cafeína. Este es un ejemplo de lo que Pellegrino plantea como la influencia de la política en la bioética. El problema de postergar soluciones a problemas evidentes que afectan a la vida y la salud, es directamente un problema bioético (16).

Tabla 1. La relación entre los principios de la bioética y la acción en cuidados paliativos

\begin{tabular}{|c|c|}
\hline Principio de bioética & Acción en cuidados paliativos \\
\hline Autonomía & $\begin{array}{l}\text { Consentimiento informado } \\
\text { Voluntad anticipada } \\
\text { Órdenes de no resucitación }\end{array}$ \\
\hline No maleficencia & $\begin{array}{l}\text { Limitación/adecuación del esfuerzo terapéutico } \\
\text { Evitar distanasia }\end{array}$ \\
\hline Beneficencia & $\begin{array}{l}\text { Ortotanasia } \\
\text { Muerte digna } \\
\text { Dar malas noticias }\end{array}$ \\
\hline Justicia & $\begin{array}{l}\text { Distribución de recursos escasos } \\
\text { Triaje } \\
\text { Vulnerabilidad }\end{array}$ \\
\hline Solidaridad & $\begin{array}{l}\text { Acompañamiento } \\
\text { Atención a la familia del enfermo } \\
\text { Despedida del enfermo }\end{array}$ \\
\hline No abandono & Apoyo y atención al enfermo respetando sus preferencias \\
\hline Doble efecto & Sedación paliativa \\
\hline
\end{tabular}

Fuente: Elaboración propia. 
En general, la bioética clínica, tal vez la rama más conocida de esta disciplina, se enfrenta a dilemas del encuentro clínico individual y a enfermedades de poblaciones y grupos. Durante la pandemia se ha visto enfrentada a considerar las obligaciones hacia los grupos sociales, cambiando el enfoque del individualismo al de satisfacer las necesidades de toda la población (18).

Desde el punto de vista poblacional, los análisis bioéticos deben considerar la extensión, dirección, distribución de los recursos, incluyendo los temas de escasez, y la problemática de los individuos vulnerables.

Los principios bioéticos para desarrollar en situaciones de problemas humanitarios son el respeto a la persona, la no maleficencia, la beneficencia, la justicia, la solidaridad, el no abandono y el principio de doble efecto (18).

En nombre del respeto a la persona, se considera fundamental considerar la autonomía. Derivados del principio de autonomía tenemos el proceso del consentimiento informado, la voluntad anticipada y las órdenes de no resucitar (19). Se habla mucho de autonomía en la pandemia. Para algunos autores, en casos de crisis extremas como en la pandemia actual, podría omitirse la decisión del paciente. Para otros, respetar la autonomía equivale al respeto de la dignidad de la persona, siempre y cuando la petición sea proporcionada, esté dentro de la legalidad y exista el recurso. Es indispensable contar con el consentimiento del enfermo para su tratamiento y conocer anticipadamente sus preferencias. Es importante platicar con el paciente acerca del final de su vida cuando él no pueda decidir y orientarlo acerca de lo que es posible y lo que no, así como de los costos emocionales y económicos que supondrán para sus familias. La información completa dada al paciente y a la familia es una obligación del médico para que la toma de decisiones sea informada (20).

La no maleficencia puede corresponder a la necesidad de adecuar el esfuerzo terapéutico, o limitar el esfuerzo terapéutico con el objetivo de no dar tratamientos fútiles a los enfermos y no caer en distanasia. La distanasia se refiere al esfuerzo fútil del médico por 
prolongar la vida del paciente, o hacer más lenta la muerte con la consiguiente angustia y sufrimiento del paciente y su familia (21). Para evitar la distanasia es importante conocer el término de adecuación del esfuerzo terapéutico o la limitación del esfuerzo terapéutico. Éste es un proceso que proporciona el tratamiento al estado del paciente. Es necesario valorar el estado clínico de un enfermo y tomar la decisión basada en evidencias médicas, para no instalar un tratamiento o para suspender uno ya iniciado. En el caso de la pandemia, adecuar el esfuerzo terapéutico será retirar al enfermo de la Unidad de Terapia Intensiva, descontinuar la ventilación artificial, renunciar a la medicación múltiple, como sucede en pacientes que han adquirido una infección nosocomial y reciben varios medicamentos cuando presentan una falla orgánica múltiple. El paciente contará en todo momento con ayuda médica, paliación de su enfermedad y acompañamiento. La relación del médico y la familia deberá ser lo más cercana posible, y se debe informar de toda medida que se tome con el enfermo (22).

La beneficencia se relaciona con el control de los síntomas y con favorecer la ortotanasia, que es sinónimo de muerte digna. Ésta es la forma de morir que prefiere la bioética y la legislación en México, y se refiere a respetar el momento de la muerte natural del paciente, sin alargar ni acortar la vida (21). Es indispensable saber dar malas noticias (24) y acompañar y favorecer el duelo, ya que es parte del final de la vida (23).

La justicia tiene que ver con la necesidad de asignar correctamente los recursos escasos, sobre todo aquellos que son no divisibles. Maximizar el bien para la mayor cantidad de pacientes es una de las metas a lograr en situaciones de crisis sanitaria. La meta es la preocupación por dar a todos una oportunidad, no discriminar y abandonar los prejuicios injustos como son el estatus, la edad y la raza. Ello significa aplicar bien el triaje, apegado a las evidencias médicas, siguiendo los modelos de predicción y sin preferencias (25); cuidar a la persona vulnerable y ofrecerle oportunidades (26) (27). 
Solidaridad es entender el difícil escenario que se presenta ante el aislamiento y procurar el acompañamiento a través de los medios posibles para el enfermo y su familia. Solidaridad se refiere a pararse junto al doliente. Requiere acción y verdadera preocupación por los demás. La despedida es una situación particularmente compleja, ya que el paciente y su familia no tienen la oportunidad de decirse adiós, ni de seguir los ritos culturales y familiares del final de la vida (28).

No abandono es un principio fundamental en medio de la crisis sanitaria, que se refiere a no dejar de atender a ningún paciente. Es indispensable que el paciente que rechaza algún tratamiento propuesto por el médico tenga la certeza de que no será desatendido por el personal de salud; es esencial evitar la falta de esperanza, sea cual sea la situación del paciente (29).

El principio de doble efecto puede estar presente en decisiones como la sedación paliativa. La intención de la sedación paliativa radica en disminuir los síntomas refractarios del paciente. Los tres más importantes son: disnea, dolor y delirio. Existe la posibilidad de que la sedación resulte en un final de la vida con un enfermo dormido, que no haya una despedida. Otra posibilidad que debe advertirse a los familiares es la posibilidad de una falla respiratoria no reversible. Cuando el paciente está en posibilidad de decidir lo hará él y, si no lo está, se pedirá a los familiares consentimiento informado. Aunque hay muchos autores que no están a favor de este procedimiento, cuando el ventilador es una medida fútil o extraordinaria, la sedación paliativa es la alternativa (30).

\section{Conclusiones}

En una época de crisis global, que pone en peligro la estabilidad sanitaria, social y económica del planeta, es necesario reflexionar para cambiar la dirección en la que va la vida de los seres humanos. 
La pandemia de Covid-19 en México: el papel fundamental de los cuidados...

La enfermedad Covid-19 apareció en diciembre de 2019 y ha puesto de manifiesto que son posibles los cambios radicales en la forma de vida. El aislamiento social, el cambio de modelo en el trabajo, en la educación, en la forma de atender los servicios sanitarios, dejan ver que el ser humano es resiliente. Asimismo, deja salir a la superficie las deudas de los estados con sus ciudadanos como, por ejemplo, que los servicios de salud son insuficientes. Es entendible que una crisis sanitaria global ponga a prueba a los servicios de salud más eficientes. En México, estas insuficiencias se han agravado por el atraso en la cobertura de la salud, la falta de atención a la epidemia de obesidad, la insuficiente inversión en actualizar los centros hospitalarios y por la poca atención a las nuevas necesidades de atención a la población, tanto en modelos de paliación como de curación.

La medicina actual cuenta con otras herramientas inscritas en la legislación, poco desarrolladas en la práctica: los cuidados paliativos y la bioética. En un marco de necesidad y escasez, se empodera la paliación apoyada en los principios bioéticos, y se hace evidente la necesidad de desarrollar estos conocimientos e impulsarlos con un presupuesto mayor, que se destine a la formación de personal sanitario, al desarrollo de unidades hospitalarias con cuidados paliativos y a la atención primaria en domicilio.

A un año del inicio de la pandemia, hay muchas preguntas por responder, muchas evidencias que descubrir, muchas cifras por revelar. El surgimiento de una nueva enfermedad infecciosa y contagiosa en un planeta con casi ocho mil millones de personas ha sido un reto, y debe ser también un motor de cambio a favor del cuidado de la vida, de la salud y de la casa común de todas las vidas.

\section{Referencias bibliográficas}

1. Shereen MA, Khan S, Kazmi A, Bashir N, Siddique R. Covid-19 infection: Origin, transmission and characteristics of human coronaviruses. J Adv Res. 2020; 24: 91-8. https://doi.org/10.1016/j.jare.2020.03.005 
2. Yan R, Zhang $Y$, Li Y, Xia L, Guo Y, Zhou Q. Structural basis for the recognition of Sars-CoV-2 by full-length human ACE2. Science (80). 2020; 367(6485): 1444-8. https://doi.org/10.1126/science.abb2762

3. Reina J. Las pandemias de la gripe. Rev Española Quimioter. 2009; 22(1): 2-4.

4. Emanuele Nicastri NP, Bartoli TA, Luciana Lepore AM, Fabrizio Palmieri GD, Luisa Marchioni SM, Giuseppe Ippolito AA. National Institute for the Infectious Diseases «L. Spallanzani», IRCCS. Recommendations for Covid-19 clinical management. Infect Dis Rep. 2020; 12: 8543. https://doi.org/10.4081/idr.2020.8543

5 . Milicevic N. The hospice movement: History and current worldwide situation. Arch Oncol. 2002; 10(1): 29-31.

6. González C, Méndez J, Romero JI, Bustamante J, Castro R, Jiménez M. Cuidados paliativos en México. Palliative care in Mexico. Rev Med Hosp Gen Méx. 2012; 75(3): 173-9.

7. Covarrubias Gómez A, Otero Lamas DM, Templos Esteban DLA, Soto Pérez de Celis E, Internacional G. Educación continua en cuidados paliativos. 2019; 42(2): 122-8.

8. Del Río Ml, Palma A. Cuidados paliativos: historia y desarrollo. Boletín Esc Med UC, Pontificia Univ. Católica Chile. 2007; 32(1): 16-22. Recuperado en: http:// cuidadospaliativos.org/uploads/2013/10/historia de CP.pdf

9. Radbruch L, De Lima L, Knaul F, Wenk R, Ali Z, Bhatnaghar S, et al. Redefining palliative care. A new consensus-based definition. J Pain Symptom Manage. 2020. Recuperado en: https://doi.org/10.1016/j.jpainsymman.2020.04.027

10. Feder SL, Akgün KM, Schulman-Green D. Palliative care strategies offer guidance to clinicians and comfort for covid-19 patient and families. Hear Lung. 2020; 49(3): 227-8. https://doi.org/10.1016/j.hrtlng.2020.04.001

11. Powell VD, Silveira MJ. What should palliative care's response be to the Covid-19 pandemic? J Pain Symptom Manage. 2020; 60(1): e1-3. https://doi.org/10. 1016/j.jpainsymman.2020.03.013

12. Hernández MACO, Rujillo ALFE. La soledat dels pacients amb Covid-19 al final de les seves. Revista de Bioética y Derecho Perspectivas Bioéticas. 2020; 5887(c): 81-98. https://doi.org/10.1344/rbd2020.50.31683

13. The Lancet. Palliative care and the Covid-19 pandemic. Lancet. 2020; 395 (10231): 1168. http://dx.doi.org/10.1016/S0140-6736 (20)30822-9

14. Reich WT. Encyclopedia of bioethics. New York; London: Macmillan Pub. Co. Simon \& Schuster Macmillan/Prentice Hall International; 1995.

15. Zalaquett R. 50 años de trasplante de corazón: la operación que enmudeció al mundo y cambió para siempre el concepto de muerte TT. 50 years after the first heart transplant: the operation that stunned the world and forever changed the concept of death. Rev chil cardiol. 2017; 36(3): 275-82. Recuperado en: http:// www.scielo.cl/scielo.php?script=sci_arttext\&pid=S0718-85602017000 300275 https://doi.org/10.4067/s0718-85602017000300275

16. Pellegrino ED. Bioethics and politics: «Doing ethics» in the public square. $J$ Med Philos. 2006; 31(6): 569-84. 
La pandemia de Covid-19 en México: el papel fundamental de los cuidados...

17. Hernández Medina MS. Overview of the overweight and obesity epidemic in Mexico. Panorama de la epidemia de sobrepeso y obesidad en México. 2020; 8(16): 65-71.

18. Wynne KJ, Petrova M, Coghlan R. Dying individuals and suffering populations: Applying a population-level bioethics lens to palliative care in humanitarian contexts: Before, during and after the Covid-19 pandemic. J Med Ethics. 2020; 46(8): 514-25. https://doi.org/10.1136/medethics-2019-105943

19. Fried TR, Bradley EH, Towle VR, Allore H. Understanding the treatment preferences of seriously ill patients. N Engl J Med. 2002; 346(14): 1061-6. https://doi. org/10.1056/nejmsa012528

20. Kirchhoffer DG. Dignity, autonomy, and allocation of scarce medical resources During Covid-19. J Bioeth Inq. 2020. https://doi.org/10.1007/s11673-020-09 998-3

21. Biondo CA, Da Silva MJP, Secco LMD. Dysthanasia, euthanasia, orthotanasia: the perceptions of nurses working in intensive care units and care implications. Rev Lat Am Enfermagem. 2009; 17(5): 613-9. https://doi.org/10.1590/s0104-1169 2009000500003

22. Pérez Pérez FM. Adecuación del esfuerzo terapéutico, una estrategia al final de la vida. The suitability of therapeutic effort: An end-of-life strategy. Semergen. 2016; 42(8): 566-74. Recuperado en: www.elsevier.es/semergen https://doi.org/ 10.1016/j.semerg.2015.11.006

23. Wallace CL, Wladkowski SP, Gibson A, White P. Grief During the Covid-19 pandemic: Considerations for palliative care providers. J Pain Symptom Manage. 2020; 60(1): e70-6. https://doi.org/10.1016/j.jpainsymman.2020.04.012

24. Reilly MAO. Effective Difficult Conversations: A Step-by-step guide. In review: Effective difficult conversations. 2020; 26(1). https://doi.org/10.5860/rusq.56. $4.297 a$

25. Xia H, Zhang W, Tu C, Kang L, Wu W, Qu Z, et al. Development of predictive nomogram for ICU mortality in severe Covid-19. SSRN Electron J. 2020. https:// doi.org/10.2139/ssrn.3627282

26. Silva DS. Ventilators by lottery: the least unjust form of allocation in the coronavirus disease 2019 pandemic. Chest. 2020; 158(3): 890-1. https://doi.org/ 10.1016/j.chest.2020.04.049

27. Powell T, Bellin E, Ehrlich AR. Older Adults and Covid-19: The most vulnerable, the hardest hit. Hastings Cent Rep. 2020; 50(3): 61-3. https://doi.org/10.1002/ hast.1136

28. Johnson SB. Advancing global health equity in the Covid-19 response: beyond solidarity. J Bioeth Inq. 2020 (April).

29. Robinson S, Kissane DW, Brooker J, Burney S. A Review of the construct of demoralization: history, definitions, and future directions for palliative care. $A m J$ Hosp Palliat Med. 2016; 33(1): 93-101. https://doi.org/10.1177/1049909114553461 30. Riisfeldt TD. Weakening the ethical distinction between euthanasia, palliative opioid use and palliative sedation. J Med Ethics. 2019; 45(2): 125-30. https:// doi.org/10.1136/medethics-2018-105074

Medicina y Ética - Enero-Marzo 2021 - Vol. 32 - Núm. 1 
